\title{
Platinum Complexes with Picoline-functionalized Benzimidazolin-2-ylidene Ligands
}

\author{
Mareike C. Jahnke, Tania Pape, and F. Ekkehardt Hahn \\ Institut für Anorganische und Analytische Chemie Westfälische Wilhelms-Universität Münster, \\ Corrensstraße 30, 48149 Münster, Germany \\ Reprint requests to Prof. Dr. F. E. Hahn. Fax: +49 251 8333108. E-mail: fehahn@ uni-muenster.de \\ Z. Naturforsch. 2010, 65b, $341-346$; received December 9, 2009 \\ Dedicated to Professor Rolf W. Saalfrank on the occasion of his $70^{\text {th }}$ birthday \\ The dicarbene platinum complexes of the type $\left[\mathrm{Pt}(\mathrm{L})_{2}\right] \mathrm{Br}_{2}[5] \mathrm{Br}_{2}-[8] \mathrm{Br}_{2}\left(\mathrm{~L}=N\right.$-alkyl- $N^{\prime}-$ \\ picolylbenzimidazolin-2-ylidene) have been prepared by two different methods. The in situ depro- \\ tonation of picoline-functionalized benzimidazolium salts $\mathbf{1}-\mathbf{4}$ with platinum acetylacetonate gave \\ the platinum complexes [5] $\mathrm{Br}_{2}-[\mathbf{8}] \mathrm{Br}_{2}$ in good yields. Complex $[\mathbf{8}] \mathrm{Br}_{2}$ has also been obtained by a \\ ligand transfer reaction from the silver dicarbene complex [9][ $\left.\mathrm{AgBr}_{2}\right]$. Attempts to cystallize $[8] \mathrm{Br}_{2}$ \\ obtained from the carbene transfer reaction led to the isolation of the previously undetected mono- \\ carbene complex $\left[\mathrm{Pt}(\mathrm{Cl})_{2} \mathrm{~L}\right](\mathbf{1 0})$ which contains only one picoline-functionalized carbene ligand \\ coordinating in a chelating fashion to the metal center.
}

Key words: Heterocyclic Carbene Complexes, Platinum(II) Complexes

\section{Introduction}

An impressive number of stable $\mathrm{N}$-heterocyclic carbenes (NHCs) and their metal complexes [1] have been prepared after the first isolation of a stable $\mathrm{N}$ heterocyclic carbene by Arduengo et al. [2]. This development is driven by the application of carbene complexes in various homogeneous catalytic reactions [3] and based on the superior donor properties of NHCs compared to phosphines leading to quite stable metal carbon bonds [1]. Most of the NHC complexes contain the ubiquitous and easily accessible imidazolin2 -ylidenes as carbene ligands [1,2]. The number of complexes bearing NHCs with an alternative topology, like the benzimidazolin-2-ylidenes, is much smaller. Benzimidazolin-2-ylidenes are also excellent ligands, exhibiting a versatile coordination chemistry. Regarding their donor properties and propensity for dimerization they assume an intermediate position between the saturated imidazolidin-2-ylidenes and the unsaturated imidazolin-2-ylidenes $[1,4]$.

There are several established procedures for the synthesis of complexes bearing benzimidazolin-2-ylidene ligands. Such complexes can be prepared by the reaction of transition metal complexes with the free carbene ligand $[4 \mathrm{a}, 5]$ or by cleavage of the $\mathrm{C}=\mathrm{C}$ dou- ble bond of dibenzotetraazafulvalenes with coordinatively unsaturated transition metal complexes $[4 \mathrm{~b}, 6]$. The most common method for the synthesis of complexes with benzimidazolin-2-ylidene ligands is the in situ deprotonation of the benzimidazolium salts followed by complex formation. Suitable transition metal complexes with basic ligands for this procedure are $\left[\mathrm{Pd}(\mathrm{OAc})_{2}\right][7],\left[\mathrm{Ni}(\mathrm{OAc})_{2}\right][8]$ or $[\mathrm{Ir}(\mu-\mathrm{OMe})(\mathrm{cod})]_{2}$ [9]. The in situ deprotonation of azolium salts with $\mathrm{Ag}_{2} \mathrm{O}$ [10] has received special interest since the silver complexes obtained in this reaction are very useful agents for carbene transfer reactions to other transition metals [11]. In addition, complexes with benzannulated N-heterocyclic carbene ligands can be prepared by a template-controlled intramolecular cyclization reaction of coordinated 2-aminophenyl isocyanides followed by $N, N^{\prime}$-dialkylation of the intermediate $\mathrm{NH}, \mathrm{NH}$-stabilized benzannulated carbene ligands [12].

We have studied the coordination chemistry of benzimidazolin-2-ylidenes and the catalytic applications of complexes with this type of NHC ligand. Special emphasis has been placed on complexes with donorfunctionalized benzimidazolin-2-ylidenes. As a result of these studies, we and others described the synthesis and properties of palladium complexes bearing functionalized bidentate [10b, 13] or pincer-type benzimid- 
azolin-2-ylidene ligands [14]. In this contribution we present the synthesis of platinum complexes bearing picoline-functionalized benzimidazolin-2-ylidenes obtained from the corresponding benzimidazolium salts by in situ deprotonation or by the carbene transfer reaction from the silver complexes.

\section{Results and Discussion}

The synthesis of picoline-functionalized benzimidazolium bromides like $\mathbf{1 - 4}$ has been described [13]. The platinum complexes $[\mathbf{5}] \mathrm{Br}_{2}-[\mathbf{8}] \mathrm{Br}_{2}$ were prepared from the benzimidazolium bromides and platinum acetylacetonate as metal precursor which leads to in situ deprotonation followed by coordination of the resulting NHC ligands to the metal center (Scheme 1) [15]. Complexes [5] $\mathrm{Br}_{2}-[\mathbf{8}] \mathrm{Br}_{2}$ were obtained as bright-yellow compounds in good (about $80 \%$ ) yields.

The platinum complexes [5] $\mathrm{Br}_{2}-[\mathbf{8}] \mathrm{Br}_{2}$ have been fully characterized by ${ }^{1} \mathrm{H}$ - and ${ }^{13} \mathrm{C}-\mathrm{NMR}$ spectroscopy, mass spectrometry and elemental analysis. The characteristic signal for the benzimidazolium C2-proton observed in the ${ }^{1} \mathrm{H}-\mathrm{NMR}$ spectra of compounds $\mathbf{1}-\mathbf{4}$ in the range $\delta_{\mathrm{H}}=9.90-10.14 \mathrm{ppm}$ [13] is absent in the spectra of complexes $[\mathbf{5}] \mathrm{Br}_{2}-[\mathbf{8}] \mathrm{Br}_{2}$. In addition, the resonances for the carbene carbon atoms in the complexes have been observed around $\delta_{\mathrm{C}}=149 \mathrm{ppm}$. The resonance for the NCN carbon atom in complexes $[6] \mathrm{Br}_{2}-[8] \mathrm{Br}_{2}$ is slightly shifted downfield compared to the parent benzimidazolium salts $\mathbf{1}-\mathbf{4}\left(\delta_{\mathrm{C}}=143.8-\right.$ $143.1 \mathrm{ppm})$ [13]. The chemical shifts for the carbene carbon atom fall in the range previously reported for platinum complexes with benzimidazolin-2-ylidene ligands $\left(\delta_{\mathrm{C}} \approx 160-145 \mathrm{ppm}\right)[10 \mathrm{~b}, 16]$ and are similar to the chemical shifts observed for platinum com-

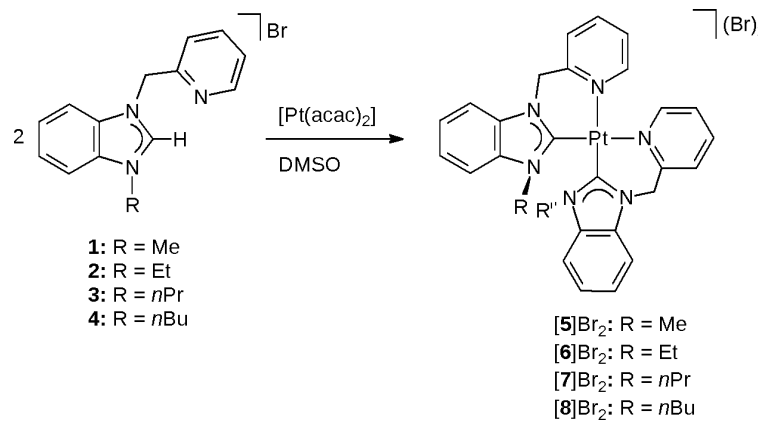

Scheme 1. Synthesis of dicarbene platinum complexes $[\mathbf{5}] \mathrm{Br}_{2}-[\mathbf{8}] \mathrm{Br}_{2}$ with picoline-functionalized benzimidazolin2-ylidene ligands. plexes with imidazolin-2-ylidene ligands $\left(\delta_{\mathrm{C}}=149.6-\right.$ $143.0 \mathrm{ppm})[15,17]$. The resonances for the protons of the methylene bridge have been observed as doublets at $\delta_{\mathrm{H}} \approx 6.50$ and $\delta_{\mathrm{H}} \approx 6.20 \mathrm{ppm}$ with a ${ }^{2} J$ coupling constant of $15.5 \mathrm{~Hz}$. This typical value for a geminal coupling $[13,14 \mathrm{c}, 15,17]$ reflects the diastereotopic nature of the two methylene protons. Contrary to the observations made for the analogous palladium complexes with picoline-functionalized benzimidazolin-2-ylidene ligands [13], only one set of signals has been detected in the ${ }^{13} \mathrm{C}$-NMR spectra for the carbon atoms of the picoline donor function. The corresponding palladium complexes of type $\left[\mathrm{PdL}_{2}\right] \mathrm{Br}_{2}$ exhibit two sets of signals due to fluxional behavior of the picoline donor functions. Apparently, the picoline donors in [5] $\mathrm{Br}_{2}-$ $[8] \mathrm{Br}_{2}$ are more tightly bound to the platinum atom thus preventing fluxional behavior.

In an alternative synthetic procedure, the preparation of the platinum complex $[\mathbf{8}] \mathrm{Br}_{2}$ was performed by a ligand transfer reaction from the corresponding silver carbene complex [9][ $\left.\mathrm{AgBr}_{2}\right]$ obtained by treatment of the benzimidazolium salt 4 with silver oxide under exclusion of light (Scheme 2). The intermediate silver complex [9] $\left[\mathrm{AgBr}_{2}\right]$ was obtained in good yield and has been fully characterized.

Carbene transfer from [9] $\left.\mathrm{AgBr}_{2}\right]$ to the platinum precursor $\left[\mathrm{PtCl}_{2}(\mathrm{NCPh})_{2}\right]$ was performed in the presence of an excess of sodium bromide to achieve complete halide exchange at the metal center. Surprisingly, the yield of the platinum complex $[8] \mathrm{Br}_{2}$ was only $54.1 \%$ compared to $85.9 \%$ obtained from the in situ deprotonation reaction (Scheme 1). The analytical data

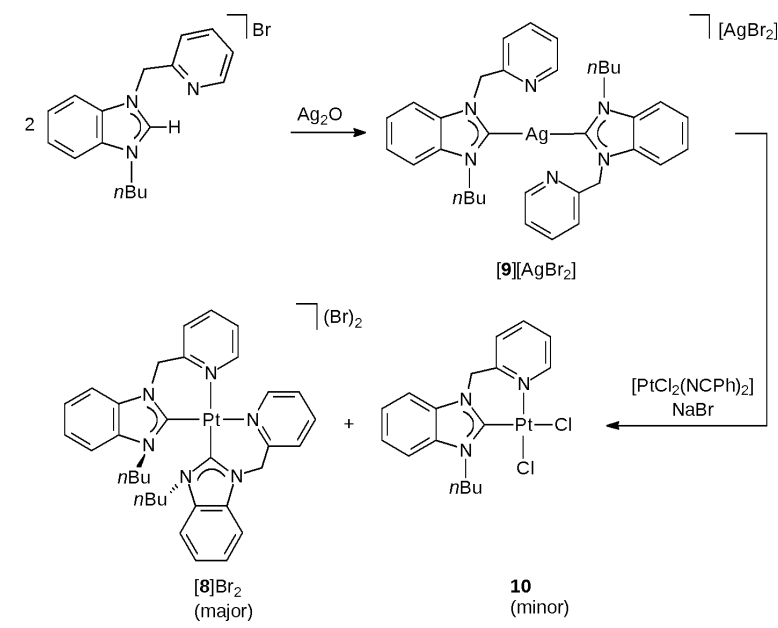

Scheme 2. Formation of platinum complex $[8] \mathrm{Br}_{2}$ and byproduct 10. 


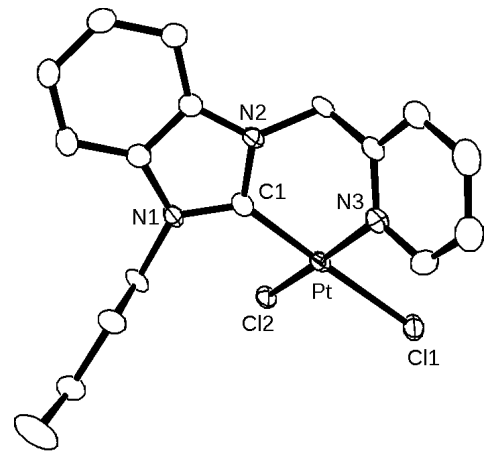

Fig. 1. Molecular structure of $\mathbf{1 0}$ (hydrogen atoms omitted for clarity, displacement ellipsoids at the $50 \%$ probability level). Selected bond lengths (A) and angles (deg): Pt-Cl1 2.3644(12), Pt-Cl2 2.3117(11), Pt-N3 2.039(4), PtC1 1.951(4), C1-N1 1.355(5), C1-N2 1.359(5); Cl1-PtCl2 88.69(4), Cl1-Pt-N3 90.64(12), Cl1-Pt-C1 178.29(13), Cl2-Pt-N3 174.82(11), Cl2-Pt-C1 93.01(13), N3-Pt-C1 87.6(2), N1-C1-N2 106.2(4).

for $[\mathbf{8}] \mathrm{Br}_{2}$ obtained by ligand transfer from the silver complex $[9]\left[\mathrm{AgBr}_{2}\right]$ are virtually identical to those obtained for the same complex synthesized by in situ deprotonation (Scheme 1), indicating in both cases coordination of two carbene ligands to the metal center.

The reaction product obtained from the ligand transfer reaction was crystallized from dichloromethane. The X-ray diffraction structure analysis with crystals obtained in this way showed surprisingly that the neutral monocarbene platinum complex $\mathbf{1 0}$ had been obtained (Fig. 1). This by-product apparently was formed already during the synthesis of $[8] \mathrm{Br}_{2}$, even though it was not detected by NMR spectroscopy or mass spectrometry prior to the crystallization experiments. The NMR spectroscopic data of $\mathbf{1 0}$ (which could not be determined) appear to be sufficiently similar to those of $[\mathbf{8}] \mathrm{Br}_{2}$ to prevent detection of $\mathbf{1 0}$. The microanalytical data of $[\mathbf{8}] \mathrm{Br}_{2}$ and $\mathbf{1 0}$ are different but not different enough to clearly indicate a contamination of $[8] \mathrm{Br}_{2}$ with the small amount of $\mathbf{1 0}$ which was subsequently isolated by crystallization.

The $\mathrm{Pt}-\mathrm{C}_{\text {carbene }}$ bond length in $\mathbf{1 0}$ (1.951(4) $\mathrm{A}$ ) compares well to equivalent distances previously observed for platinum complexes with benzimidazolin-2-ylidene ligands (1.941(11)-2.015(4) ^̊) [16] or platinum complexes bearing methylene-bridged bis(imidazolin2-ylidene) ligands (Pt-C $\mathrm{C}_{\text {carbene }} 1.942(8)-1.991(3) \AA$ ) $[15,17]$. Due to the smaller atomic radius of platinum(II) compared to palladium(II), a shorter Pt$\mathrm{N}_{\text {picoline }}$ bond length (Pt-N3 2.039(4) $\AA$ ) is observed in 10 compared to the $\mathrm{Pd}-\mathrm{N}_{\text {picoline }}$ bond lengths found in the palladium complexes bearing picolinefunctionalized benzimidazolin-2-ylidene ligands ( $\mathrm{Pd}-$ $\mathrm{N}_{\text {picoline }}$ 2.082(3)-2.144(4) $\AA$ ) [13]. As a consequence of the stronger trans-influence of the benzimidazolin-2-ylidene donor function the $\mathrm{Pt}-\mathrm{Cl1}$ bond $(2.3644(12) \AA)$ is elongated compared to the PtCl2 bond (2.3117(11) $\AA$ ). Complex 10 shows only a slight deviation from the ideal square-planar geometry with the angle $\mathrm{Cl} 2-\mathrm{Pt}-\mathrm{N} 3\left(174.82(11)^{\circ}\right)$ exhibiting the largest deviation from linearity. The bite angle of the picoline-functionalized carbene ligand (N3-Pt-C1 $\left.87.6(2)^{\circ}\right)$ is larger than the corresponding angles found in the analogous palladium complexes $\left(\mathrm{C}_{\text {carbene }}-\mathrm{Pd}-\right.$ $\mathrm{N}_{\text {picoline }} 84.93(13)-86.82(13)^{\circ}$ ) [13].

We have described the in situ deprotonation of picoline-functionalized benzimidazolium salts with $\left[\mathrm{Pt}(\mathrm{acac})_{2}\right]$ leading to carbene platinum(II) complexes with bidentately coordinated picoline-functionalized benzimidazolin-2-ylidene ligands. These complexes are also accessible by ligand transfer reaction from the corresponding silver complexes. Use of $\left[\mathrm{PtCl}_{2}(\mathrm{NCPh})_{2}\right]$ as the platinum source leads to the formation of the monocarbene complex $\mathbf{1 0}$ as a side product.

\section{Experimental Section}

Chemicals and solvents were purchased from Aldrich. NMR spectra were recorded on a Bruker AC 200 spectrometer. MALDI mass spectra were obtained with a Varian MAT 212 spectrometer. Elemental analyses were performed with a Vario EL III CHNS Elemental Analyzer at the Institut für Anorganische und Analytische Chemie, Westfälische Wilhelms-Universität Münster. The picoline-functionalized benzimidazolium bromides $\mathbf{1}-\mathbf{4}$ were prepared according to published procedures [13].

\section{General procedure for the preparation of the platinum complexes by in situ deprotonation}

2 eq. of one of the $N$-alkyl- $N^{\prime}$-picolylbenzimidazolium bromides $1-4(1.0 \mathrm{mmol})$ and 1 eq. of platinum acetylacetonate $(0.197 \mathrm{~g}, 0.5 \mathrm{mmol})$ were dissolved in DMSO $(10 \mathrm{~mL})$. The reaction mixture was stirred for $2 \mathrm{~h}$ at ambient temperature, subsequently heated up to $50{ }^{\circ} \mathrm{C}$ for $16 \mathrm{~h}$, and finally stirred for $3 \mathrm{~h}$ at $125^{\circ} \mathrm{C}$. The solvent was removed in vacuo, and the obtained bright-yellow residue was dissolved in a small amount of methanol. This solution was slowly added while stirring to $200 \mathrm{~mL}$ of ice-cold diethyl ether. Complexes $[\mathbf{5}] \mathrm{Br}_{2}-[\mathbf{8}] \mathrm{Br}_{2}$ precipitated and were isolated by filtration. Drying in vacuo gave the complexes as bright-yellow solids. 
[Bis(N-methyl- $N^{\prime}$-picolylbenzimidazolin-2-ylidene)platinum] dibromide ([5]Br $)$

Yield: 0.314 g $(0.39 \mathrm{mmol}, 78.3 \%) . \quad-{ }^{1} \mathrm{H}$ NMR (200.1 MHz, [D 6 DMSO, ppm): $\delta=8.52\left(\mathrm{~d},{ }^{3} J=5.5 \mathrm{~Hz}\right.$, $2 \mathrm{H}$, pyridine- $\left.\mathrm{H}_{\varepsilon}\right), 8.38-8.25(\mathrm{~m}, 6 \mathrm{H}$, Ar- $\mathrm{H}$, pyridine- $\mathrm{H})$, $7.92-7.82\left(\mathrm{~d},{ }^{3} \mathrm{~J}=7.8 \mathrm{~Hz}, 2 \mathrm{H}, \mathrm{Ar}-\mathrm{H}\right), 7.73-7.40(\mathrm{~m}, 6 \mathrm{H}$, Ar-H, pyridine-H), $6.53\left(\mathrm{~d},{ }^{2} \mathrm{~J}=15.5 \mathrm{~Hz}, 2 \mathrm{H}, \mathrm{N}_{-} \mathrm{CH}_{2}-\right.$ pyridine), $6.26\left(\mathrm{~d},{ }^{2} \mathrm{~J}=15.5 \mathrm{~Hz}, 2 \mathrm{H}, \mathrm{N}-\mathrm{CH}_{2}\right.$-pyridine), 4.13 $\left(\mathrm{s}, 6 \mathrm{H}, \mathrm{NCH}_{3}\right) .-{ }^{13} \mathrm{C} \mathrm{NMR}\left(50.3 \mathrm{MHz},\left[\mathrm{D}_{6}\right] \mathrm{DMSO}, \mathrm{ppm}\right)$ : $\delta=154.0$ (pyridine- $\mathrm{C}_{\alpha}$ ), 153.2 (pyridine- $\left.\mathrm{C}_{\varepsilon}\right), 141.1$ (pyridine- $\mathrm{C}_{\gamma}$ ), 132.9, 132.4, 126.0, 125.7 (Ar-C), 124.5, 123.9 (pyridine- $\mathrm{C}_{\beta, \delta}$ ), 112.2, 111.8 (Ar-C), $51.2\left({\mathrm{~N}-\mathrm{CH}_{2}-}^{-}\right.$ pyridine), $33.6\left(\mathrm{NCH}_{3}\right)$. The resonance for the carbene carbon atom was not observed. - MS (MALDI-TOF): $\mathrm{m} / \mathrm{z}=721$ $[\mathrm{M}-\mathrm{Br}]^{+}$. - Elemental analysis for $\mathrm{C}_{28} \mathrm{H}_{26} \mathrm{~N}_{6} \mathrm{Br}_{2} \mathrm{Pt}$ (801.4): calcd. C 41.96, H 3.27, N 10.49; found C 41.57, H 2.97, N 10.31 .

[Bis(N-ethyl-N'-picolylbenzimidazolin-2-ylidene)platinum]dibromide $\left([\mathbf{6}] \mathrm{Br}_{2}\right)$

Yield: $0.337 \mathrm{~g}$ (0.41 mmol, 81.2\%). $\quad-{ }^{1} \mathrm{H}$ NMR (200.1 MHz, [D 6 ]DMSO, ppm): $\delta=8.56\left(\mathrm{~d},{ }^{3} J=5.5 \mathrm{~Hz}, 2 \mathrm{H}\right.$, pyridine- $\left.\mathrm{H}_{\varepsilon}\right), 8.38-8.25(\mathrm{~m}, 6 \mathrm{H}, \mathrm{Ar}-\mathrm{H}$, pyridine- $\mathrm{H}), 7.92-$ $7.81(\mathrm{~m}, 2 \mathrm{H}, \mathrm{Ar}-\mathrm{H}), 7.73-7.38(\mathrm{~m}, 6 \mathrm{H}, \mathrm{Ar}-\mathrm{H}$, pyridine-H), $6.48\left(\mathrm{~d},{ }^{2} \mathrm{~J}=15.5 \mathrm{~Hz}, 2 \mathrm{H}, \mathrm{N}-\mathrm{CH}_{2}\right.$-pyridine $), 6.21(\mathrm{~d}$, ${ }^{2} J=15.5 \mathrm{~Hz}, 2 \mathrm{H}, \mathrm{N}-\mathrm{CH}_{2}$-pyridine), $4.43-4.18(\mathrm{~m}, 2 \mathrm{H}$, $\left.\mathrm{NCH}_{2} \mathrm{CH}_{3}\right), 3.86-3.59\left(\mathrm{~m}, 2 \mathrm{H}, \mathrm{NCH}_{2} \mathrm{CH}_{3}\right), 1.16(\mathrm{t}, 6 \mathrm{H}$, $\left.\mathrm{NCH}_{2} \mathrm{CH}_{3}\right) .-{ }^{13} \mathrm{C} \mathrm{NMR}\left(50.3 \mathrm{MHz},\left[\mathrm{D}_{6}\right] \mathrm{DMSO}, \mathrm{ppm}\right)$ : $\delta=154.1$ (pyridine- $\mathrm{C}_{\alpha}$ ), 153.0 (pyridine- $\mathrm{C}_{\varepsilon}$ ), $149.5(\mathrm{NCN})$, 141.0 (pyridine- $\mathrm{C}_{\gamma}$ ), 132.8, 132.0, 126.2, 125.9 (Ar-C), 124.5, 123.9 (pyridine- $\mathrm{C}_{\beta, \delta}$ ), 112.4, 112.0 (Ar-C), 51.3 (N-CH - -pyridine), $43.2\left(\mathrm{NCH}_{2} \mathrm{CH}_{3}\right), 14.1\left(\mathrm{NCH}_{2} \mathrm{CH}_{3}\right)$. MS (MALDI-TOF): $\mathrm{m} / \mathrm{z}=749[\mathrm{M}-\mathrm{Br}]^{+}$. - Elemental analysis for $\mathrm{C}_{30} \mathrm{H}_{30} \mathrm{~N}_{6} \mathrm{Br}_{2} \mathrm{Pt}$ (829.5): calcd. C 43.44, H 3.65, N 10.13; found C 43.21, H 3.46, N 9.94.

[Bis(N-propyl- $N^{\prime}$-picolylbenzimidazolin-2-ylidene)-

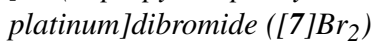

Yield: 0.356 g $(0.42 \mathrm{mmol}, 83.1 \%) . \quad-{ }^{1} \mathrm{H}$ NMR (200.1 MHz, [D 6 ]DMSO, ppm): $\delta=8.56\left(\mathrm{~d},{ }^{3} J=5.5 \mathrm{~Hz}\right.$, $2 \mathrm{H}$, pyridine- $\mathrm{H}), 8.38-8.25(\mathrm{~m}, 6 \mathrm{H}$, Ar- $\mathrm{H}$, pyridine- $\mathrm{H})$, $7.87\left(\mathrm{~d},{ }^{3} \mathrm{~J}=7.8 \mathrm{~Hz}, 2 \mathrm{H}, \mathrm{Ar}-\mathrm{H}\right), 7.73-7.40$ (m, 6H, Ar-H, pyridine-H), $6.55\left(\mathrm{~d},{ }^{2} \mathrm{~J}=15.5 \mathrm{~Hz}, 2 \mathrm{H}, \mathrm{N}-\mathrm{CH}_{2}\right.$-pyridine), $6.18\left(\mathrm{~d},{ }^{2} \mathrm{~J}=15.5 \mathrm{~Hz}, 2 \mathrm{H}, \mathrm{N}-\mathrm{CH}_{2}\right.$-pyridine), $4.38-4.19$ (m, $\left.2 \mathrm{H}, \mathrm{NCH}_{2} \mathrm{CH}_{2} \mathrm{CH}_{3}\right), 3.61-3.39\left(\mathrm{~m}, 2 \mathrm{H}, \mathrm{NCH}_{2} \mathrm{CH}_{2} \mathrm{CH}_{3}\right)$, 1.97-1.71 (m, 2H, $\left.\mathrm{NCH}_{2} \mathrm{CH}_{2} \mathrm{CH}_{3}\right), 1.66-1.43$ (m, $\left.2 \mathrm{H}, \mathrm{NCH}_{2} \mathrm{CH}_{2} \mathrm{CH}_{3}\right), 0.53$ (t, $\left.6 \mathrm{H}, \mathrm{NCH}_{2} \mathrm{CH}_{2} \mathrm{CH}_{3}\right)$. ${ }^{13} \mathrm{C}$ NMR (50.3 MHz, [D 6 DMSO, ppm): $\delta=154.2$ (pyridine- $\mathrm{C}_{\alpha}$ ), 153.1 (pyridine- $\mathrm{C}_{\varepsilon}$ ), $149.5(\mathrm{NCN}), 140.3$ (pyridine- $\mathrm{C}_{\gamma}$ ), 133.2, 132.5, 127.0, 126.8 (Ar-C), 124.0, 123.6 (pyridine- $\mathrm{C}_{\beta, \delta}$ ), 112.3, $111.9(\mathrm{Ar}-\mathrm{C}), 51.0\left(\mathrm{~N}^{-\mathrm{CH}_{2}-}\right.$ pyridine), $49.7\left(\mathrm{NCH}_{2} \mathrm{CH}_{2} \mathrm{CH}_{3}\right), 22.5\left(\mathrm{NCH}_{2} \mathrm{CH}_{2} \mathrm{CH}_{3}\right)$, $11.4\left(\mathrm{NCH}_{2} \mathrm{CH}_{2} \mathrm{CH}_{3}\right)$. - MS (MALDI-TOF): $\mathrm{m} / \mathrm{z}=777$ $[\mathrm{M}-\mathrm{Br}]^{+}$. - Elemental analysis for $\mathrm{C}_{32} \mathrm{H}_{34} \mathrm{~N}_{6} \mathrm{Br}_{2} \mathrm{Pt}$ (857.5): calcd. C 44.82, H 4.00, N 9.80; found C 44.51, H 3.74, N 9.35.

[Bis(N-butyl- $N^{\prime}$-picolylbenzimidazolin-2-ylidene)platinum]dibromide $\left([8] \mathrm{Br}_{2}\right)$

Yield: $0.380 \mathrm{~g}(0.43 \mathrm{mmol}, 85.9 \%) . \quad-{ }^{1} \mathrm{H}$ NMR (200.1 MHz, [D 6 ]DMSO, ppm): $\delta=8.54\left(\mathrm{~d},{ }^{3} J=5.5 \mathrm{~Hz}\right.$, $2 \mathrm{H}$, pyridine- $\mathrm{H}), 8.32-8.18(\mathrm{~m}, 6 \mathrm{H}, \mathrm{Ar}-\mathrm{H}), 7.87(\mathrm{~d}$, $\left.{ }^{3} J=8.2 \mathrm{~Hz}, 2 \mathrm{H}, \mathrm{Ar}-\mathrm{H}\right), 7.69-7.61(\mathrm{~m}, 4 \mathrm{H}$, pyridine-H), $7.57\left(\mathrm{~d},{ }^{3} \mathrm{~J}=5.4 \mathrm{~Hz}, 2 \mathrm{H}\right.$, pyridine-H), $6.51\left(\mathrm{~d},{ }^{2} \mathrm{~J}=\right.$ $15.5 \mathrm{~Hz}, 2 \mathrm{H}, \mathrm{N}-\mathrm{CH}_{2}$-pyridine), $6.24\left(\mathrm{~d},{ }^{2} \mathrm{~J}=15.5 \mathrm{~Hz}, 2 \mathrm{H}\right.$, $\mathrm{N}-\mathrm{CH}_{2}$-pyridine), $4.41-4.21\left(\mathrm{~m}, 2 \mathrm{H}, \mathrm{NCH}_{2} \mathrm{CH}_{2} \mathrm{CH}_{2} \mathrm{CH}_{3}\right)$, $3.75-3.53\left(\mathrm{~m}, \quad 2 \mathrm{H}, \quad \mathrm{NCH}_{2} \mathrm{CH}_{2} \mathrm{CH}_{2} \mathrm{CH}_{3}\right), \quad 1.92-1.67$ $\left(\mathrm{m}, 2 \mathrm{H}, \quad \mathrm{NCH}_{2} \mathrm{CH}_{2} \mathrm{CH}_{2} \mathrm{CH}_{3}\right), \quad 1.43-1.21 \quad(\mathrm{~m}, 2 \mathrm{H}$, $\left.\mathrm{NCH}_{2} \mathrm{CH}_{2} \mathrm{CH}_{2} \mathrm{CH}_{3}\right), \quad 1.07-0.89$ (m, $4 \mathrm{H}, \quad \mathrm{NCH}_{2} \mathrm{CH}_{2}-$ $\left.\mathrm{CH}_{2} \mathrm{CH}_{3}\right), 0.53\left(\mathrm{t}, 6 \mathrm{H}, \mathrm{NCH}_{2} \mathrm{CH}_{2} \mathrm{CH}_{2} \mathrm{CH}_{3}\right),-{ }^{13} \mathrm{C} \mathrm{NMR}$ (50.3 MHz, [D $\left.\left.{ }_{6}\right] \mathrm{DMSO}, \mathrm{ppm}\right): \delta=154.3$ (pyridine- $\mathrm{C}_{\alpha}$ ), 153.2 (pyridine- $\mathrm{C}_{\varepsilon}$ ), $149.6(\mathrm{NCN}), 140.4$ (pyridine- $\mathrm{C}_{\gamma}$ ), 133.2, 132.5, 127.0, 126.8 (Ar-C), 124.0, 123.6 (pyridine$\mathrm{C}_{\beta, \delta}$ ), 112.3, 111.9 (Ar-C), $51.1\left(\mathrm{~N}_{-} \mathrm{CH}_{2}\right.$-pyridine), 48.8 $\left(\mathrm{NCH}_{2} \mathrm{CH}_{2} \mathrm{CH}_{2} \mathrm{CH}_{3}\right), \quad 30.8\left(\mathrm{NCH}_{2} \mathrm{CH}_{2} \mathrm{CH}_{2} \mathrm{CH}_{3}\right), \quad 19.7$ $\left(\mathrm{NCH}_{2} \mathrm{CH}_{2} \mathrm{CH}_{2} \mathrm{CH}_{3}\right), 13.6\left(\mathrm{NCH}_{2} \mathrm{CH}_{2} \mathrm{CH}_{2} \mathrm{CH}_{3}\right)$. - MS (MALDI-TOF): $\mathrm{m} / \mathrm{z}=805[\mathrm{M}-\mathrm{Br}]^{+}$. - Elemental analysis for $\mathrm{C}_{34} \mathrm{H}_{38} \mathrm{~N}_{6} \mathrm{Br}_{2} \mathrm{Pt}$ (885.6): calcd. C 46.11, H 4.33, N 9.49; found $\mathrm{C} 45.78, \mathrm{H} 4.02, \mathrm{~N} 9.13$.

\section{[Bis(N-butyl-N'-picolylbenzimidazolin-2-ylidene)silver]- [dibromoargentate] ([9][AgBr $]$ )}

$1.0 \mathrm{mmol}$ of $N$-butyl- $N$-picolylbenzimidazolium bromide $4(0.346 \mathrm{~g})$ and $1.1 \mathrm{mmol}$ of silver oxide $(0.255 \mathrm{~g})$ were suspended in dichloromethane $(60 \mathrm{~mL})$. The reaction mixture was stirred under exclusion of light for $12 \mathrm{~h}$ at ambient temperature. The resulting suspension was filtered through Celite ${ }^{\circledR}$, and the filtrate was brought to dryness. The residue was dissolved in dichloromethane $(5 \mathrm{~mL})$ and added dropwise to $200 \mathrm{~mL}$ of diethyl ether. The precipitate obtained was collected and dried in vacuo.

Yield: $0.392 \mathrm{~g}$ (0.43 mmol, 86.7\%). $-{ }^{1} \mathrm{H}$ NMR $\left(200.1 \mathrm{MHz},\left[\mathrm{D}_{6}\right] \mathrm{DMSO}, \mathrm{ppm}\right): \delta=8.48(\mathrm{~d}, 2 \mathrm{H}$, pyridine- $\left.\mathrm{H}_{\varepsilon}\right), \quad 7.90-7.76(\mathrm{~m}, \quad 6 \mathrm{H}, \quad$ Ar- $\mathrm{H}), \quad 7.52-7.39$ $\left(\mathrm{m}, \quad 6 \mathrm{H}, \quad \mathrm{Ar}-\mathrm{H}, \quad\right.$ pyridine- $\left.\mathrm{H}_{\gamma}\right), 7.33-7.24(\mathrm{~m}, \quad 4 \mathrm{H}$, pyridine- $\mathrm{H}_{\beta}$, pyridine- $\left.\mathrm{H}_{\delta}\right), 5.86\left(\mathrm{~s}, \mathrm{br}, 4 \mathrm{H}, \quad \mathrm{N}-\mathrm{CH}_{2}-\right.$ pyridine), 4.49 (t, $\left.4 \mathrm{H}, \quad \mathrm{NCH}_{2} \mathrm{CH}_{2} \mathrm{CH}_{2} \mathrm{CH}_{3}\right), 1.89-1.80$ (m, $\left.4 \mathrm{H}, \mathrm{NCH}_{2} \mathrm{CH}_{2} \mathrm{CH}_{2} \mathrm{CH}_{3}\right), 1.41-1.22\left(\mathrm{~m}, 4 \mathrm{H}, \mathrm{NCH}_{2}-\right.$ $\left.\mathrm{CH}_{2} \mathrm{CH}_{2} \mathrm{CH}_{3}\right), \quad 0.87 \quad\left(\mathrm{t}, \quad 6 \mathrm{H}, \quad \mathrm{NCH}_{2} \mathrm{CH}_{2} \mathrm{CH}_{2} \mathrm{CH}_{3}\right)$. ${ }^{13} \mathrm{C}$ NMR (50.3 MHz, [D 6 ]DMSO, ppm): $\delta=190.1$ $(\mathrm{NCN}), 155.3$ (pyridine- $\mathrm{C}_{\alpha}$ ), 149.4 (pyridine- $\left.\mathrm{C}_{\varepsilon}\right), 137.2$ (pyridine- $\mathrm{C}_{\gamma}$ ), 133.5, 133.2 (Ar-C), 123.9 (pyridine- $\mathrm{C}_{\beta}$ ), 123.8 (pyridine- $\mathrm{C}_{\delta}$ ), 123.1, 122.0, 112.2, 112.0 (Ar-C), 
53.1 (N-CH -pyridine), $48.3\left(\mathrm{NCH}_{2} \mathrm{CH}_{2} \mathrm{CH}_{2} \mathrm{CH}_{3}\right), 31.9$ $\left(\mathrm{NCH}_{2} \mathrm{CH}_{2} \mathrm{CH}_{2} \mathrm{CH}_{3}\right), \quad 19.3\left(\mathrm{NCH}_{2} \mathrm{CH}_{2} \mathrm{CH}_{2} \mathrm{CH}_{3}\right), \quad 13.5$ $\left(\mathrm{NCH}_{2} \mathrm{CH}_{2} \mathrm{CH}_{2} \mathrm{CH}_{3}\right.$ ). - MS (MALDI): $\mathrm{m} / z=639,637$ $\left(\left[\mathrm{M}-\mathrm{AgBr}_{2}\right]^{+}\right)$. - Elemental analysis for $\mathrm{C}_{34} \mathrm{H}_{38} \mathrm{~N}_{6} \mathrm{Br}_{2} \mathrm{Ag}_{2}$ (906.3): calcd. C 45.06, H 4.23, N 9.27; found C 44.78, $\mathrm{H} 4.01, \mathrm{~N} 9.13$.

\section{Transmetalation leading to $[8] \mathrm{Br}_{2}$ and $\mathbf{1 0}$}

1.0 eq. of the silver complex $[9]\left[\mathrm{AgBr}_{2}\right](0.227 \mathrm{~g}$, $0.25 \mathrm{mmol}), 1.0$ eq. of $\left[\mathrm{Pt}(\mathrm{Cl})_{2}(\mathrm{NCPh})_{2}\right](0.118 \mathrm{~g}$, $0.25 \mathrm{mmol})$ and an excess of $\mathrm{NaBr}(0.5 \mathrm{~g}, 5.0 \mathrm{mmol})$ were suspended in dichloromethane $(50 \mathrm{~mL})$. The reaction mixture was stirred for $12 \mathrm{~h}$ und subsequently filtered through Celite ${ }^{\circledR}$. The filtrate was brought to dryness. Work-up proceeded as described for complexes $[\mathbf{5}] \mathrm{Br}_{2}-[\mathbf{8}] \mathrm{Br}_{2}$. A yellow solid consisting of $[\mathbf{8}] \mathrm{Br}_{2}$ and a small amount of $\mathbf{1 0}$ was isolated. Yield: $0.120 \mathrm{~g}(0.14 \mathrm{mmol}, 54.1 \%$, relative to $\left.[8] \mathrm{Br}_{2}\right)$. The spectroscopic and microanalytical data of the reaction product are virtually identical to those recorded for $[8] \mathrm{Br}_{2}$ obtained via the in situ deprotonation reaction described above.

\section{X-Ray structure determination of complex [10]}

A suitable crystal of $\mathbf{1 0}$ was mounted on a Bruker AXS 2000 CCD diffractometer equipped with a rotating anode us- ing $\operatorname{Mo} K_{\alpha}$ radiation $(\lambda=0.71073 \AA)$ and a CCD area detector. Diffraction data were measured at $153(2) \mathrm{K}$ in the range $3.8 \leq 2 \theta \leq 60.1^{\circ}$. Structure solution [18] and refinement [19] were achieved with standard Patterson and Fourier techniques, respectively. All non-hydrogen atoms were refined with anisotropic displacement parameters. Hydrogen atoms were added to the structure model in calculated positions.

Selected crystallographic data for 10: Formula $\mathrm{C}_{17} \mathrm{H}_{19} \mathrm{~N}_{3} \mathrm{Cl}_{2} \mathrm{Pt}, M=531.34$, pale-yellow crystal, $0.14 \times$ $0.11 \times 0.10 \mathrm{~mm}^{3}$, triclinic, space group $P \overline{1}, Z=2, a=$ 8.6033(13), $b=9.6660(15), c=10.605(2) \AA, \alpha=87.336(3)$, $\beta=81.507(3), \gamma=86.371(3)^{\circ}, V=869.8(2) \AA^{3}, \rho_{\text {calcd }}=$ $2.03 \mathrm{~g} \mathrm{~cm}^{-3}, \mu=8.4 \mathrm{~mm}^{-1}$, empirical absorption correction $(0.3868 \leq T \leq 0.4881), 9997$ intensities collected $( \pm h, \pm k$, $\pm l), 4974$ independent $\left(R_{\text {int }}=0.0288\right)$ and 4530 observed intensities $[I \geq 2 \sigma(I)], 209$ refined parameters, residuals for all data $R=0.0387, w R 2=0.0802$, residual electron density

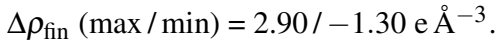

CCDC 757791 contains the supplementary crystallographic data for this paper. These data can be obtained free of charge from The Cambridge Crystallographic Data Centre via www.ccdc.cam.ac.uk/data_request/cif.

\section{Acknowledgement}

Financial support by the Deutsche Forschungsgemeinschaft (SFB 424 and IRTG 1444) is gratefully acknowledged.
[1] a) F. E. Hahn, Dalton Trans. 2009, 6893, an issue dedicated to recent developments of the organometallic chemistry of NHC ligands; b) F. E. Hahn, M. C. Jahnke, Angew. Chem. 2008, 120, 3166; Angew. Chem. Int. Ed. 2008, 47, 3122; c) F.E. Hahn, Angew. Chem. 2006, 118, 1374; Angew. Chem. Int. Ed. 2006, 45, 1348; d) D. Bourissou, O. Guerret, F. P. Gabbaï, G. Bertrand, Chem. Rev. 2000, 100, 39.

[2] A. J. Arduengo III, R. L. Harlow, M. Kline, J. Am. Chem. Soc. 1991, 113, 361.

[3] a) A. T. Normand, K. J. Cavell, Eur. J. Inorg. Chem. 2008, 2781; b) R. Coberán, E. Mas-Marzá, E. Peris, Eur. J. Inorg. Chem. 2009, 1700; c) M. Poyatos, J. A. Mata, E. Peris, Chem. Rev. 2009, 109, 3677.

[4] a) F.E. Hahn, L. Wittenbecher, R. Boese, D. Bläser, Chem. Eur. J. 1999, 5, 1931; b) F. E. Hahn, L. Wittenbecher, D. Le Van, R. Fröhlich, Angew. Chem. 2000, 112, 551; Angew. Chem. Int. Ed. 2000, 39, 541.

[5] F. E. Hahn, T. von Fehren, R. Fröhlich, Z. Naturforsch. 2004, $59 b, 348$

[6] a) F.E. Hahn, T. von Fehren, L. Wittenbecher, R. Fröhlich, Z. Naturforsch. 2004, 59b, 541; b) F.E. Hahn, T. von Fehren, L. Wittenbecher, R. Fröhlich, Z. Naturforsch. 2004, 59b, 544; c) F. E. Hahn, T. von Fehren, T. Lügger, Inorg. Chim. Acta 2005, 358, 4137; d) E. Çetinkaya, P. B. Hitchcock, H. Küçükbay, M. F.
Lappert, S. Al-Juaid, J. Organomet. Chem. 1994, 481, 89.

[7] a) F. E. Hahn, C. Holtgrewe, T. Pape, Z. Naturforsch. 2004, 59b, 1051; c) H. Türkmen, T. Pape, F.E. Hahn, B. Çetinkaya, Eur. J. Inorg. Chem. 2009, 285; c) F. E. Hahn, M. Foth, J. Organomet. Chem. 1999, 585, 241.

[8] a) H. V. Huynh, C. Holtgrewe, T. Pape, L. L. Koh, F. E. Hahn, Organometallics 2006, 25, 245; b) H. V. Huynh, R. Jothibasu, Eur. J. Inorg. Chem. 2009, 1926.

[9] a) F.E. Hahn, C. Holtgrewe, T. Pape, M. Martin, E. Sola, L. A. Oro, Organometallics 2005, 24, 2203; b) H. Türkmen, T. Pape, F. E. Hahn, B. Çetinkaya, Eur. J. Inorg. Chem. 2008, 5418.

[10] a) H. M. J. Wang, I. J. B. Lin, Organometallics 1998, 17, 972; b) F.E. Hahn, M.C. Jahnke, T. Pape, Organometallics 2006, 25, 5927; c) M.C. Jahnke, J. Paley, F. Hupka, J. J. Weigand, F. E. Hahn, Z. Naturforsch. 2009, 64b, 1458.

[11] For recent reviews on carbene transfer reactions from silver carbene complexes see: a) J.C. Garrison, W. J. Youngs, Chem. Rev. 2005, 105, 3978; b) I. J. B. Lin, C. S. Vasam, Coord. Chem. Rev. 2007, 251, 642; c) J.C. Y. Lin, R. T.W. Huang, C.S. Lee, A. Bhattacharyya, W. S. Hwang, I. J. B. Lin, Chem. Rev. 2009, 109,3561 . 
[12] a) M. Tamm, F.E. Hahn, Coord. Chem. Rev. 1999, 182,175 ; b) F.E. Hahn, V. Langenhahn, N. Meier, T. Lügger, W.P. Fehlhammer, Chem. Eur. J. 2003, 9, 704; c) F.E. Hahn, C. García Plumed, M. Münder, T. Lügger, Chem. Eur. J. 2004, 10, 6285; d) F.E. Hahn, V. Langenhahn, T. Lügger, T. Pape, D. Le Van, Angew. Chem. 2005, 117, 3825; Angew. Chem. Int. Ed. 2005, 44, 3759; e) O. Kaufhold, A. Flores-Figueroa, T. Pape, F.E. Hahn, Organometallics 2009, 28, 896; f) A. Flores-Figueroa, O. Kaufhold, K.-O. Feldmann, F. E. Hahn, Dalton Trans. 2009, 9334.

[13] M. C. Jahnke, T. Pape, F. E. Hahn, Eur. J. Inorg. Chem. 2009, 1960

[14] a) F.E. Hahn, M.C. Jahnke, V. Gomez-Benitez, D. Morales-Morales, T. Pape, Organometallics 2005, 24, 6458; b) M. C. Jahnke, T. Pape, F. E. Hahn, Z. Naturforsch. 2007, 62b, 357; c) F. E. Hahn, M.C. Jahnke, T. Pape, Organometallics 2007, 26, 150; d) T. Tu, X. Bao, W. Assenmacher, H. Peterlik, J. Daniels, K. H. Dötz, Chem. Eur. J. 2009, 15, 1853; e) T. Tu, J. Malineni, K. H. Dötz, Adv. Synth. Catal. 2008, 350, 1791; f) H. V. Huynh, D. Yuan, Y. Han, Dalton Trans. 2009, 7262 .
[15] a) S. Ahrens, E. Herdtweck, S. Goutal, T. Strassner, Eur. J. Inorg. Chem. 2006, 1268; b) S. Ahrens, T. Strassner, Inorg. Chim. Acta 2006, 359, 4789.

[16] a) Y. Han, H. V. Huynh, G. K. Tan, Organometallics 2007, 26, 4612; b) A. J. Boydston, J.D. Rice, M. D. Sanderson, O.L. Dykhno, C.W. Bielawski, Organometallics 2006, 25, 6087.

[17] a) M. Muehlhofer, T. Strassner, E. Herdtweck, W. A. Herrmann, J. Organomet. Chem. 2002, 660, 121; b) C.A. Quezada, J.C. Garisson, C.A. Tessier, W. J. Youngs, J. Organomet. Chem. 2003, 671, 183; c) A. Meyer, M. A. Taige, T. Strassner, J. Organomet. Chem. 2009, 694, 1861; d) C. Chen, H. Qiu, W. Chen, D. Wang, J. Organomet. Chem. 2008, 693, 3273.

[18] G. M. Sheldrick, SHELXS-97, Program for the Solution of Crystal Structures, University of Göttingen, Göttingen (Germany) 1997. See also: G. M. Sheldrick, Acta Crystallogr. 1990, A46, 467.

[19] G. M. Sheldrick, SHELXL-97, Program for the Refinement of Crystal Structures, University of Göttingen, Göttingen (Germany) 1997. See also: G. M. Sheldrick, Acta Crystallogr. 2008, A64, 112. 